\title{
Online Signature Verification Based on Legendre Series Representation. Consistency Analysis of Different Feature Combinations
}

\author{
Marianela Parodi and Juan Carlos Gómez \\ Laboratory for System Dynamics and Signal Processing, \\ FCEIA, Universidad Nacional de Rosario, CIFASIS, CONICET \\ \{parodi,gomez\}@cifasis-conicet.gov .ar
}

\begin{abstract}
In this paper, orthogonal polynomial series are used to approximate the time functions associated to the signatures and the coefficients in these series are used as features to model them. A novel consistency factor is proposed to quantify the discriminative power of different combinations of time functions related to the signing process. Pen coordinates, incremental variation of pen coordinates and pen pressure are analyzed for two different signature styles, namely, Western signatures and Chinese signatures from a publicly available Signature Database. Two state-of-the-art classifiers, namely, Support Vector Machines and Random Forests are used in the verification experiments. The obtained error rates are comparable to results reported over the same signature datasets in a recent signature verification competition.
\end{abstract}

Keywords: Online Signature Verification, Legendre Polynomials, Consistency Factor.

\section{Introduction}

Signature verification is the most popular method for identity verification. It is a non-invasive biometric technique, and people are familiar with the use of signatures for identity verification in their everyday life.

Electronic pen-input devices, such as digitizer tablets and PDAs, are gaining popularity for signature acquisition in several daily applications, increasing the interest in online signature verification. In online systems, the signature is parameterized by different discrete time functions, e.g., pen coordinates, pressure and inclination angles. Researchers have long argued about the effectiveness of these different time functions for verification purposes. During the First International Signature Verification Competition (SVC2004), the results using only pen coordinates outperformed those adding pen pressure and inclination angles [1. Since then, several works have been presented concerning the best set of features to model the signatures. A desirable property for any feature is that it must have high consistency. The feature values of the genuine signatures should be close to each other while the ones of genuine and forged signatures should be not. A consistency model is introduced in [2, where several features are compared. Pen coordinates and some derived features proved to be the most reliable 
ones. In [3], the authors assert that using pen coordinates leads to better results than adding pen pressure. On the other hand, some works showed improvements when combining pen coordinates with pen pressure and inclination angles 4]. The conflicting results observed in the literature and the lack of a widely used consistency model, make the discussion still open. Moreover, most of the works do not consider the cultural origin of the signatures.

The main contributions of this paper are the following:

- A new feature extraction approach based on Legendre series representation of the time functions associated to the signatures is proposed. To the best of the authors' knowledge this is the first time that this approach is used in the context of signature verification.

- A novel consistency factor is proposed to quantify the discriminative power of different combinations of the time functions associated to the signing process. The pros and cons of these different combinations are analyzed.

- The experiments are performed on the most recent signature datasets, containing Western and Chinese signatures, which have been used in the latest signature verification competition. Two state-of-the-art classifiers, namely, Support Vector Machines (SVMs) and Random Forests (RFs), are used to perform the verification experiments. For the results, the EER (Equal Error Rate) and the cost of the log-likelihood ratios $\hat{C}_{l l r}$ are reported.

\section{Feature Extraction}

Several features have been proposed for online signature verification. In this paper, features composed by the orthogonal polynomial approximation coefficients of the different time functions associated to the signatures are proposed.

Under certain conditions, a function $f$ in an inner product space $H([a, b])$ can be uniquely represented by $f=\sum_{i=0}^{\infty} \alpha_{i} L_{i}$, where $\left\{L_{i}\right\}_{i=0}^{\infty}$ are orthonormal basis and $\alpha_{i}=\left\langle f, L_{i}\right\rangle$, where $\langle\cdot, \cdot\rangle$ stands for the inner product.

It is not difficult to prove that the best (in the sense of the metric induced by the inner product) approximation of $f \in H([a, b])$ in an $N$-dimensional subspace is given by

$$
f \approx \sum_{i=0}^{N} \alpha_{i} L_{i}
$$

The idea here is to approximate the time functions measured during the acquisition of the signature by a finite series expansion in orthonormal polynomials in the interval $[0,1]$, and to use their coefficients as features. Particularly, orthonormal Legendre polynomials are considered as the basis functions $L_{i}$ in this paper.

Since the time functions $f(t)$ are unknown, the coefficients in (1) cannot be computed as $\alpha_{i}=\left\langle f, L_{i}\right\rangle$, but rather they have to be estimated from a set of $M$ (usually larger than $N+1$ ) samples of the function at the time instants $\left\{t_{1}, t_{2}, \cdots, t_{M}\right\}$, resorting to least squares estimation techniques.

A similar approach to represent handwritten symbols but using function moments instead of the corresponding series expansions was presented in [5]. 


\section{Consistency Measure}

A desirable property for a feature is its consistency. Features associated to genuine signatures should be close to each other while distances between features associated to genuine and forged signatures should be relatively large. A measure of consistency based on the features would be difficult to compute since they may have different lengths. It is then more reasonable to define a consistency measure based on the distances among features and not on the features themselves. In this paper, the consistency of a given feature will be computed based on the statistics of the intraclass (for the genuine signature class) and interclass (between the genuine and forged signature classes) distances. A consistency factor $d$, for each writer, could then be defined as follows:

$$
d=\frac{\mu_{D}\left(C_{g}, C_{f}\right)-\mu_{D}\left(C_{g}, C_{g}\right)}{\sqrt{\sigma_{D}^{2}\left(C_{g}, C_{g}\right)}+\sqrt{\sigma_{D}^{2}\left(C_{g}, C_{f}\right)}},
$$

where $C_{g}$ and $C_{f}$ stand for the genuine and the forged classes, respectively, and where $\mu_{D}\left(C_{g}, C_{g}\right)$ and $\sigma_{D}^{2}\left(C_{g}, C_{g}\right)$, and $\mu_{D}\left(C_{g}, C_{f}\right)$ and $\sigma_{D}^{2}\left(C_{g}, C_{f}\right)$ are the sample means and sample variances of the genuine intraclass distances and the genuine-forged interclass distances, respectively.

The consistency factor in (2) is normalized in such a way that, under the assumption of Gaussian distributions for the involved distances, it equals 1 when $\mu_{D}\left(C_{g}, C_{f}\right)$ and $\mu_{D}\left(C_{g}, C_{g}\right)$ are separated by the sum of the respective standard deviations. This is illustrated in Fig. 1. The larger the consistency factor, the more consistent the features are. A slightly different definition for the consistency measure is presented in [2].

\section{Evaluation Protocol}

The publicly available SigComp2011 Dataset [6] is used. It has two separate datasets, one containing genuine and forged Western signatures (Dutch ones) and the other one containing genuine and forged Chinese signatures. The available forgeries are skilled forgeries, which are simulated signatures in which forgers (different writers than the reference one) are allowed to practice the reference signature for as long as they deem it necessary. The data was collected from

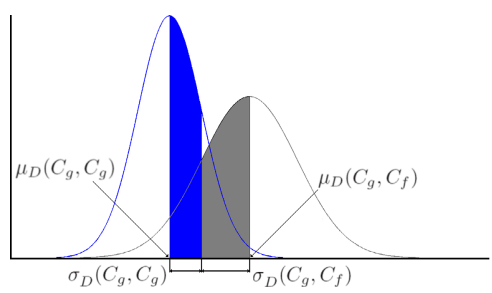

Fig. 1. Genuine intraclass distance distribution (blue) and genuine-forged interclass distance distribution (grey) 
realistic and forensically relevant scenarios. The signatures were acquired using a ballpoint pen on paper (WACOM Intuos3 A3 Wide USB Pen Tablet), which is the natural writing process.

The measured data consists of three discrete time functions: pen coordinates $x$ and $y$, and pen pressure $p$. In addition to the raw data, the incremental variation of the $x$ and $y$ pen coordinates ( $\Delta x$ and $\Delta y$, respectively) are computed. In [2] and 3] the authors list $x, y, \Delta x$ and $\Delta y$ among the most reliable features.

In [2], the most common time functions were individually compared based on a consistency model. In this paper, a novel consistency factor is proposed and several combinations of time functions (modeled by the Legendre series coefficients) are tested. In particular, the following combinations will be considered to compute their consistency factors and to assess the verification performance: $\boldsymbol{i}$. pen coordinates: $x, y ; \boldsymbol{i i}$. pen coordinates and pen pressure: $x, y, p ; \boldsymbol{i i i}$. incremental variation of pen coordinates: $\Delta x, \Delta y$; $\boldsymbol{i v}$. incremental variation of pen coordinates and pen pressure: $\Delta x, \Delta y, p ; \boldsymbol{v}$. pen coordinates and incremental variation of pen coordinates: $x, y, \Delta x, \Delta y$; $\boldsymbol{v i}$. pen coordinates, incremental variation of pen coordinates and pen pressure: $x, y, \Delta x, \Delta y, p$.

\subsection{Consistency Computation}

The consistency factor quantifies the discriminative power of a particular combination of time functions. Based on this value, it is possible to select the most suitable combination of time functions to be used in a verification system. This selection has to be done in the training stage. The consistency factor should then be computed with the signatures available during this stage. It is the common case that, when training a verification system, skilled forgeries are not available. For this reason, the consistency factor for a particular writer will be computed using the genuine signatures corresponding to all the remaining writers as forgeries (usually called random forgeries). This will result in larger consistency factors compared with the ones that would be obtained using skilled forgeries for each writer. In any case, since the used database does contain skilled forgeries, the consistency factor will also be computed using them, for comparison purposes.

\subsection{Verification Performance Evaluation}

Two well known state-of-the-art classifiers, SVMs [7] and RFs [8], are used to assess the verification performance of the different time function combinations.

The datasets in the SigComp2011 database are divided into two sets, namely, the Training Set and the Testing Set (see [6] for a detailed description). For each of the datasets, namely, Dutch and Chinese, the optimization of the metaparameters of the system is performed over the corresponding Training Set while the corresponding Testing Set is used for independent testing purposes.

The tuning parameters to adjust are the order of the Legendre polynomials 1 and the internal parameters of the classifiers. For SVMs, the parameter 2 are

\footnotetext{
${ }^{1}$ To select the optimal order, this parameter was varied from 1 to 25 .

${ }^{2}$ Optimized, within the range $10^{-10}$ to $10^{10}$, using tune.svm of the e1071 Package 9 .
} 
the scale $\sigma^{2}$ in the Radial Basis Functions (RBF) kerne 3 , and the regularization parameter $C$. For RFs, the parameters are the number of trees to grow and the number of randomly selected splitting variables to be considered at each node 4

To obtain statistically significant results, a 5 -fold cross-validation (5-fold CV) is performed over the Testing Set to estimate the testing errors. For each instance of the 5-fold CV, a signature model is trained for each writer, using only genuine signatures. To train the signature model for a particular writer, the genuine class was formed with the genuine signatures of the writer in the corresponding training set of the 5 -fold CV, while the forged class was formed with the genuine signatures of all the remaining writers in the dataset available in the same training set. The genuine and forged signatures of the writer under consideration in the corresponding testing set of the 5-fold CV are used for testing. Only skilled forgeries are considered to calculate the testing errors. Random forgeries are not considered for testing since they seldom appear in real situations.

To evaluate the performance, the EER is calculated, using the Bosaris toolkit [10], from the Detection Error TradeOff (DET) Curve as the point in the curve where the FRR (False Rejection Rate) equals the FAR (False Acceptance Rate). The cost of the log-likelihood ratios $\hat{C}_{l l r}$ and its minimal possible value $\hat{C}_{l l r}^{\text {min }}$ [10] are computed using the toolkit as well. A smaller value of $\hat{C}_{l l r}^{m i n}$ indicates a better performance of the system. Using these measurements to evaluate the performance of a signature verification system is proposed in 6], where the importance of computing the likelihood ratios was highlighted since they make Forensic Handwriting Experts (FHEs) able to combine the results obtained from an automatic verification system with other evidence presented in a court of law.

\section{Results and Discussion}

Figure 2 shows the boxplots associated to the consistency factors for each feature combination over the 54 writers for the Dutch dataset (first row) and over the 10 writers for the Chinese dataset (second row).

The consistency factors computed resorting to random and skilled forgeries lead to the same conclusion that the most consistent (largest value of the consistency factor) time function combination is $\Delta x, \Delta y, p$, for both datasets. This agreement is important since, in real situations, the verification system can be subjected to skilled forgeries but the decisions about which combination is to be used must be necessarily made based on consistency factors computed with random forgeries, since skilled forgeries are usually not available for training.

The pen pressure, when combined with the other considered time functions, improves the consistency factor of the combinations, for both datasets. In addition, the pen coordinates $x$ and $y$ are not included in the most consistent combination $(\Delta x, \Delta y, p)$. This suggests that, based on the consistency factor, using the pen coordinates $x$ and $y$ is not a good choice for any of the datasets.

\footnotetext{
3 The RBF kernel is defined as $K(\mathbf{x}(n), \mathbf{x}(k))=e^{\|\mathbf{x}(n)-\mathbf{x}(k)\|^{2} / \sigma^{2}}$.

${ }^{4}$ In general, the default values are a good choice for these parameters.
} 

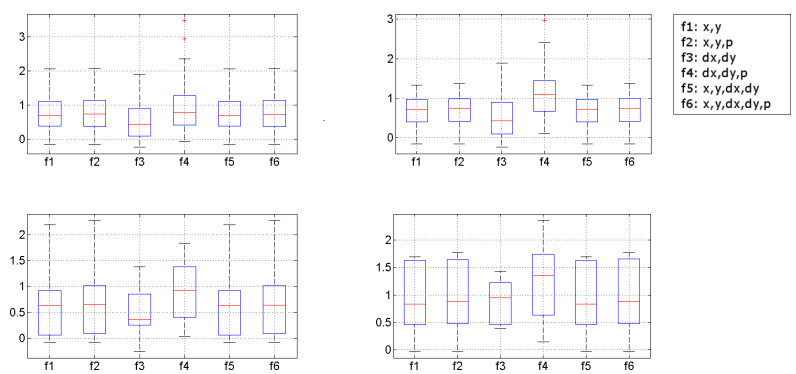

Fig. 2. Boxplots for the consistency factor: over the 54 Dutch authors, computed with skilled (top left) and random (top right) forgeries; over the 10 Chinese authors, computed with skilled (bottom left) and random (bottom right) forgeries

Table 1 presents the verification results for the Dutch and Chinese datasets 5 In addition to making the combination more consistent, incorporating the pen pressure improves the performance in most of the cases for Dutch and Chinese data, independently of the classifier being used. This is in agreement with the ideas presented in [4, where the authors stated that adding pen pressure could improved the results depending on the classification algorithm. In addition, in almost all the cases, using the computed sequences $\Delta x$ and $\Delta y$ leads to a better performance than using directly the measured sequences $x$ and $y$, which are also less consistent. These experimental results agree with the ones in [2], where the pen coordinates and the speed are listed among the most reliable features.

The results obtained with $\mathrm{RF}$ are better than those obtained with SVM. In addition, for some time functions, the results depend on the classifier being used. For Dutch data, whenever using pen coordinates together with SVMs, the performance is strongly degraded, then they are not robust against the different classifiers. For Chinese data, $x$ and $y$ coordinates appear to be more robust than $\Delta x$ and $\Delta y$, nevertheless, whenever using the pen pressure, the results improve, then pen pressure is robust against the different classifiers. The consistency factor, on the other hand, does not depend on the classifier being used. Then, it can be used to indicate the potential of a particular combination for being effective in the verification stage, independently of the classification method.

The position information is likely to be better suited, in the sense of the verification errors, for the Chinese data than for the Dutch data. Chinese signature style is, in most of the cases, close to the Chinese handwriting style, consisting of one or more multi-trace ideograms, while Western signatures can adopt several different styles. Since Chinese characters usually convey their meaning through pictorial resemblance to a physical object, it is likely that the position information has more discriminative power than in the case of Dutch data.

${ }^{5}$ The following tuning parameters were used: SVM: $\sigma^{2}=10^{7}$ and $C=1$ (Dutch dataset), and $\sigma^{2}=10^{7}$ and $C=10$ (Chinese dataset); RF: number of trees $=500$, number of randomly selected splitting variables $=\sqrt{P}$, where $P$ is the dimension of the feature vector, and Legendre polynomials: $N=21$ (both datasets). 
Table 1. Verification results for the Dutch (left) and Chinese (right) Datasets

\begin{tabular}{|c|c|c|c|c||c|c|c|}
\hline \multicolumn{2}{|c|}{} & \multicolumn{2}{|c||}{ Dutch Dataset } & \multicolumn{3}{c|}{ Chinese Dataset } \\
\hline Features & Class. & EER & $\hat{C}_{l l r}$ & $\hat{C}_{l l r}^{m i n}$ & EER & $\hat{C}_{l l r}$ & $\hat{C}_{l l r}^{m i n}$ \\
\hline \multirow{2}{*}{$x, y$} & SVM & 12.32 & 0.461 & 0.3832 & 12.67 & 0.5222 & 0.4419 \\
& RF & 8.94 & 0.3265 & 0.288 & 12.18 & 0.4587 & 0.3808 \\
\hline \multirow{2}{*}{$x, y, p$} & SVM & 12.39 & 0.4322 & 0.373 & 11.03 & 0.4164 & 0.3435 \\
& RF & 7.39 & 0.2751 & 0.2396 & 10.32 & 0.3849 & 0.3159 \\
\hline \multirow{2}{*}{$\Delta x, \Delta y$} & SVM & 8.55 & 0.3482 & 0.3032 & 14.42 & 0.5467 & 0.4458 \\
& RF & 5.92 & 0.2648 & 0.2035 & 10.8 & 0.4 & 0.3266 \\
\hline \multirow{2}{*}{$\Delta x, \Delta y, p$} & SVM & 7.63 & 0.3501 & 0.2657 & 12.74 & 0.5086 & 0.4187 \\
& RF & $\mathbf{5 . 9 1}$ & $\mathbf{0 . 2 3 7}$ & $\mathbf{0 . 1 9 5}$ & 11.09 & 0.3938 & 0.2994 \\
\hline \multirow{2}{*}{$x, y, \Delta x, \Delta y$} & SVM & 12.35 & 0.461 & 0.3833 & 13.44 & 0.5084 & 0.4418 \\
& RF & 6.46 & 0.2497 & 0.2122 & 11.83 & 0.4293 & 0.354 \\
\hline \multirow{2}{*}{$x, y, \Delta x, \Delta y, p$} & SVM & 12.54 & 0.4478 & 0.3744 & 10.8 & 0.4393 & 0.3696 \\
& RF & 7.24 & 0.2676 & 0.251 & $\mathbf{1 0 . 0 3}$ & $\mathbf{0 . 3 6}$ & $\mathbf{0 . 2 9 6 9}$ \\
\hline \hline \multicolumn{2}{|c|}{ System } & Acc. & $\hat{C}_{l l r}$ & $\hat{C}_{l l r}^{m i n}$ & Acc. & $\hat{C}_{l l r}$ & $\hat{C}_{l l r}^{m i n}$ \\
\hline \multicolumn{2}{|c|}{ commercial } & 96.27 & 0.2589 & 0.1226 & 93.17 & 0.4134 & 0.2179 \\
\hline 1 1st. non-commercial & 93.49 & 0.4928 & 0.2375 & 84.81 & 0.5651 & 0.3511 \\
\hline
\end{tabular}

The best result for the Dutch data is obtained using the $\Delta x, \Delta y, p$ combination. This makes sense since pen coordinates are not reliable for this data, and adding the pen pressure improves the results. This also confirms what the consistency factor is indicating, being $\Delta x, \Delta y, p$ the most consistent combination. The best result for the Chinese data is obtained combining all the time functions available, viz, $x, y, \Delta x, \Delta y, p$. Despite the fact that the most consistent combination is the one containing only $\Delta x, \Delta y$ and $p$, Chinese signatures appear to be more complex than Dutch ones, then it is not surprising that more features are needed to model the signature, in order to reach better verification results.

A straightforward relationship between the consistency of the features and the corresponding verification performance can be observed. For instance, adding the pen pressure results in a larger consistency factor and in a better verification performance. It was also argued that $x$ and $y$ coordinates are not consistent and results using the incremental coordinates $\Delta x$ and $\Delta y$ outperform them. This close relationship is also observed in the case of the most consistent combination for the Dutch data $(\Delta x, \Delta y, p)$ which is the best one in the sense of the verification results as well. On the other hand, the combination achieving the best verification results for the Chinese data $(x, y, \Delta x, \Delta y, p)$ is not the most consistent one. This is probably due to the complexity of this data as mentioned above.

For the purposes of comparison, the results for the best commercial and noncommercial systems in the SigComp2011 competition are included in the last two rows of Table 1 Even tough the results are not as good as the corresponding to the best commercial system (xyzmo, see [6]), they would have ranked first among the non-commercial systems and second among all the participants. Finally, the results for the Dutch signatures are better than those for the Chinese ones, 
confirming the observations in [6] and indicating that Chinese data is more challenging and that a lot of research has to be done on this type of data.

\section{Conclusions}

A novel consistency factor was proposed to quantify the discriminative power of different combinations of time functions related to the signing process. Several combinations were studied for two different signature styles, namely, Western and Chinese, of the most recent publicly available Signature Database. The proposed consistency factor proved to be well suited since it is robust against skilled forgeries and a close relationship between its value and the verification performance associated to a time function combination can be found.

The experiments showed that combining the pen pressure with the other time functions makes the combination more consistent and leads to a better verification performance for both datasets, independently of the classifier being used. In addition, in most of the cases, using $\Delta x$ and $\Delta y$ leads to a better performance than using directly $x$ and $y$, which are also less consistent.

The $\Delta x, \Delta y, p$ combination achieved the best verification performance for the Dutch data, and resulted to be the most consistent combination as well. For the Chinese data, the $x, y, \Delta x, \Delta y, p$ combination achieved the best verification results. Although this is not the most consistent combination, it is not surprising that more features are needed to model Chinese signatures since they appear to be more complex than Dutch ones.

The results obtained with RF are better than the ones obtained with SVM, for both signature styles. Nevertheless, since the consistency factor does not depend on the classifier being used, it indicates the potential of a particular combination for the verification, independently of the classification method.

The use of Legendre polynomials to model the signatures proved to be a good choice, resulting in verification performances comparable to those of other state-of-the-art verification systems, tested on the same datasets. In addition, the proposed signature model would allow for a dimensionality reduction with respect to the case of using all the points in the time functions.

\section{References}

1. Yeung, D.-Y., Chang, H., Xiong, Y., George, S.E., Kashi, R.S., Matsumoto, T., Rigoll, G.: SVC2004: First International Signature Verification Competition. In: Zhang, D., Jain, A.K. (eds.) ICBA 2004. LNCS, vol. 3072, pp. 16-22. Springer, Heidelberg (2004)

2. Lei, H., Govindaraju, V.: A Comparative Study on the Consistency of Features in On-line Signature Verification. Pattern Recogn. Lett. 147, 2483-2489 (2005)

3. Kholmatov, A., Yanikoglu, B.: Identity Authentication Using Improved Online Signature Verification Method. Pattern Recogn. Lett. 26, 2400-2408 (2005)

4. Muramatsu, D., Matsumoto, T.: Effectiveness of Pen Pressure, Azimuth, and Altitude Features for Online Signature Verification. In: Lee, S.-W., Li, S.Z. (eds.) ICB 2007. LNCS, vol. 4642, pp. 503-512. Springer, Heidelberg (2007) 
5. Golubitsky, O., Watt, S.M.: Distance-Based Classification of Handwritten Symbols. Int. Journal of Doc. Anal. and Recogn. 13, 133-146 (2010)

6. Liwicki, M., Malik, M.I., den Heuvel, C.E., Chen, X., Berger, C., Stoel, R., Blumenstein, M., Found, B.: Signature Verification Competition for Online and Offline Skilled Forgeries (SigComp 2011). In: Int. Conf. on Doc. Anal. and Recogn. (2011)

7. Vapnik, V.: The Nature of Statistical Learning Theory. Springer, NY (1995)

8. Breiman, L.: Random Forests. Technical Report, Statistics Department, University of California, Berkeley (2001)

9. Dimitriadou, E., Hornik, K., Leisch, F., Meyer, D., Weingessel, A.: Misc. Functions of the Department of Statistics (e1071). TU Wien (2010)

10. Brümmer, N., du Preez, J.: Application-Independent Evaluation of Speaker Detection. Comput. Speech and Lang. 20, 230-275 (2006) 